\title{
Identification asymmetry of parafoveal stimulus pairs
}

\author{
GARVIN CHASTAIN and LORALIE LAWSON \\ Boise State University, Boise, Idaho 83725
}

\begin{abstract}
Three experiments, involving 8,10 , and 10 naive participants, respectively, were conducted to examine the phenomenon in which the peripheral member of a parafoveally appearing stimulus pair is more accurately identified than the central. In the first experiment, the asymmetry was observed with stimulus pairs which had but one distinguishing feature; this could have implications for the notion that feature perturbations between stimuli provide the basis for the effect. The second experiment eliminated an explanation based upon acuity at the position of the midpoint of the stimulus configuration, since the asymmetry remains when this position is held constant. The involvement of response or decision factors seems unlikely, since the third experiment found the effect with blocked presentations involving responses to only one member of the pair. It is concluded that accounts which appeal to difficulties in separating stimuli from the overall pair configuration fail to explain more accurate identification of the peripheral stimulus when the pair is positioned at a constant distance from fixation.
\end{abstract}

Identification of a letter appearing parafoveally is impaired more by the presence of another letter on its peripheral side than by one on its central side. Several different explanations of this asymmetry have appeared in the literature. White (1976) collected data which he interpreted to indicate that the iconic trace of very brief presentations is scanned from the periphery inward toward fixation. With two or three letters in the array, his participants identified the target letter, which always lay 3 deg from fixation, most rapidly when it was leftmost in the left visual field and rightmost in the right visual field. However, other studies have found interference from irrelevant elements in the array when viewing time was unlimited (Taylor \& Brown, 1972; Townsend, Taylor, \& Brown, 1971), demonstrating the asymmetry in situations other than those involving iconic scanning. Banks, Bachrach, and Larson (1977) found that more spatial separation was required between the target and a peripheral letter than between the target and a central letter for identification to occur. Arrays were viewed continuously, and nontargets were moved slowly away from the target letter, beginning $1 \mathrm{sec}$ after exposure. The authors argued that the asymmetry was related to the distance from the fovea of the target plus nontarget configuration, since the midpoint of the two letters was located further from the fovea when the nontarget was peripheral than

Requests for reprints should be sent to Garvin Chastan, Department of Psychology, Boise State University, Boise, Idaho 83725. We would like to thank an anonvmous reviewer for a number of helpful suggestions, most of which were incorporated into the firial draft of the manuscript. when it was central. Observations from a more recent series of experiments, which also used accuracy criteria (Banks, Larson, \& Prinzmetal, 1979), were interpreted as supporting this explanation. Banks, Bachrach, and Larson (1979) continued to advance the hypothesis that the target and nontarget combine into a Gestalt-like configuration whose components are more difficult to distinguish as the midpoint of the cluster is moved further from the fovea. Finally, Krumhansl (Krumhansl, 1977; Krumhansl \& Thomas, 1977) has interpreted her results as indicating that perturbations of letter features occur primarily in a foveal direction, as outlined in Wolford's (1975) feature perturbation model. In this view, some features from the peripheral letter arrive at the locus of those from the central letter. While the peripheral letter may still be identified from those features which do not undergo perturbation, it is likely that the central letter will not be identified since the total feature group at its locus will differ from that possible for any one letter. This model assumes that since features of confusable letters are similar, they may be exchanged with less damage to identification than if the dissimilar features of nonconfusable letters are redistributed. The theory is essentially an extension of one, proposed by Estes, Allmeyer, and Reder (1976), which states that positional uncertainty of features increases with retinal eccentricity, and that in the face of such uncertainty, the assumed locus of a feature tends to be shifted foveally. Krumhansl and Thomas (1977), as they had predicted, found that the asymmetry was more marked for letter pairs which were nonconfusable. Stimuli for Krumhansl's studies were selected 
on the basis of Townsend's (1971) alphabetic confusion matrix, with the only definition of features being that they were shared to a greater extent by confusable than by nonconfusable letters. In consonance with the experiments of Banks, Bachrach, and Larson (1977), Krumhansl and Thomas (1977) found that the probability of an error indicating feature perturbations was not a function of stimulus duration.

The following series of experiments was conducted to contrast the explanations of the asymmetry in identification accuracy for parafoveally presented figure pairs. Each experiment involved identifying the orientation of figures consisting of only a single distinguishing feature: rotated Cs. In the first experiment, one figure always appeared at a given distance from fixation, while its flanking figure lay at one of four locations, two peripheral and two central relative to it. Asymmetric identification would be difficult to attribute to perturbation of the distinguishing feature from the peripheral to the central figure, since this should render the orientation of the former figure at least as uncertain as that of the latter. In the second experiment, the distance from fixation of both the central and peripheral figure was held constant. If the peripheral figure is better identified despite being positioned further from the fovea than the central figure, the asymmetry cannot be due to changes in the location of the midpoint of the figure pair. The final experiment required the identification of only one figure, which was flanked centrally or peripherally by a circular nontarget. Since the location of the nontarget was held constant throughout a block of trials, and only one response per presentation was required, identification asymmetry was probably not due to processing interference from decision or response factors.

\section{EXPERIMENT 1}

The contention that a figure is identified less well with a peripherally presented counterpart than with a centrally presented one due to the predominance of feature perturbations in a foveal direction (Krumhansl, 1977; Krumhansl \& Thomas, 1977) might be tested by using as stimuli figures which each have only one feature. Unfortunately, we know of no systematic attempt to define those aspects of patterns which the visual system utilizes as basic features. Nevertheless, some investigators have designated features by which letters may be distinguished. Lindsay and Norman (1972) have constructed a feature list describing each letter in the English alphabet. According to their chart, the letter $C$ has only one distinguishing feature, a discontinuous curve. Gibson (1969) describes the $C$ as having two distinguishing features, an open curve and symmetry. However, symmetry is logically a secondary relational feature, since it depends upon the orientation of the open curve (see Lupker, 1979). If the visual system responds to the discontinuous curve as a basic feature and if the feature of the $\mathrm{C}$ underwent perturbation to another spatial location, the position of the gap might no longer be apparent. Moreover, if the feature of a $\mathrm{C}$ arrived at the locus of a second $\mathrm{C}$, the position of the gap in the latter might be uncertain, depending upon the spatial position ascribed to the arriving feature. Therefore, if most feature perturbations move from the peripheral figure to the central one, identifying the gap position in the former would be at least as difficult as in the latter. The task in the present experiment was to identify the orientation of rotated Cs (gap down or gap up). Asymmetry in performance favoring the figure in the peripheral location could be interpreted to indicate that feature perturbations are not necessarily involved in the typically observed effect.

\section{Method}

Participants. Eight students served in the experiment for extra credit in a general psychology course. Each reported normal or corrected-to-normal vision, and performed in a single session lasting approximately $45 \mathrm{~min}$.

Apparatus. A Scientific Prototype Model N-1000 three-channel manual tachistoscope was used by the participants, who selftriggered stimuli with a hand-held microswitch for binocular viewing. The luminance of each channel was approximately $130.0 \mathrm{~cd} /$ $\mathrm{m}^{2}$, as measured by a Spectra Lumicon with Photospot attachment. The experiment room was dimly illuminated by a shielded 40-W bulb.

Stimuli. The masking array consisted of a row of 12 equally spaced dollar signs, with a small fixation dot between the innermost two. The array subtended a horizontal visual angle of $7.454 \mathrm{deg}$ and a vertical angle of $.910 \mathrm{deg}$. Each dollar sign measured $.375 \mathrm{deg}$ wide and $.910 \mathrm{deg}$ high, and intercharacter spaces were $.268 \mathrm{deg}$ in width. The Cs were rotated $90 \mathrm{deg}$ clockwise or counterclockwise, and each $C$ subtended a visual angle of $.535 \mathrm{deg}$ horizontally and $.375 \mathrm{deg}$ vertically. A duplicate of one such $\mathbf{C}$ is shown in the upright position in Figure 1. A C never replaced either of the two outermost dollar signs in the masking array. Each $C$ was positioned such that the slash of a dollar sign would completely cover the gap if both were shown simultaneously. All stimuli were drawn on white index cards with black ink and were mounted on Masonite slides. A Pilot Razor Point pen was used to trace the figures from a Timesaver Lettering Guide No. 760-V. Gaps left by the stencil supports were carefully filled in manually by a work-study student unfamiliar with the experimental hypothesis. A sample of the displays for the left visual field presentation is shown in Table 1; those in the right visual field were mirror images. The two rotated $\mathrm{Cs}$

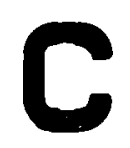

Figure 1. The stimulus figure in its upright orientation. 
Table 1

Positioning of Stimuli for Experiment 1

\begin{tabular}{|c|c|c|c|c|c|c|c|c|c|c|c|c|}
\hline \multirow{2}{*}{$\begin{array}{c}\text { Position of } \\
\text { Variable } \\
\text { Figure }\end{array}$} & \multirow{2}{*}{\multicolumn{2}{|c|}{$\begin{array}{c}\text { Separa- } \\
\text { tion }\end{array}$}} & \multicolumn{10}{|c|}{ Retinal Location } \\
\hline & & & 5 & 4 & 3 & 2 & 11 & 2 & 3 & 4 & 5 & \\
\hline Peripheral & 2 & & $\omega$ & & $\Omega$ & & & & & & & \\
\hline Peripheral & 1 & & & $\omega$ & $\omega$ & & & & & & & \\
\hline Central & 2 & & & & $n$ & & $\Omega$ & & & & & \\
\hline Central & 1 & & & & $n$ & $\omega$ & & & & & & \\
\hline \multicolumn{2}{|c|}{ Pre-and Postmask } & $\$$ & $\$$ & $\$$ & $\$$ & $\$$ & $\$ \cdot \$$ & $\$$ & $\$$ & $\$$ & $\$$ & $\$$ \\
\hline
\end{tabular}

appearing on each trial were on different slides, which were illuminated simultaneously for $100 \mathrm{msec}$, preceded and followed by the masking array. The resultıng loss of contrast for the Cs would have made the dollar signs much more distinct than the stimulus pair; this was corrected by overlapping the masking array with seven layers of Scotch Magic Tape. Contrast between figure and ground for the stimulus and masking arrays therefore appeared approximately equal.

Design. Ninety-six criterion trials were given each participant, with stimulus pairs appearing in each visual field on half the trials. One figure always appeared in Position 3, as shown in Table 1, with another figure appearing in one of Positions 1, 2, 4 , and 5 equally often. Orientation of the $C$ (clockwise or counterclockwise rotation) was varied factorially. All variables were randomized across the presentation sequence with the constraint that each possible stimulus display was shown once within each block of 32 trials. The sequence was presented in forward order to half the participants and in reverse order to the remainder.

Procedure. The participant was familiarized with the possible locations and the possible orientations of the stimulus figures, which werc described as flattened circles. The instructions were to look at the central fixation dot and initiate each presentation with the microswitch after the experimenter had given a brief verbal ready signal to indicate that all slides were in place. After a presentation, the orientation of each figure was identified by reporting the position of its gap aloud, using the words "upper" and "lower," and aiways reporting the leftmost figure first. The word "blank" was to be reported in place of the gap position if all information was absent for a particular figure, although guessing was encouraged if any information had been extracted. Sixteen practice trials preceded the 96 criterion trials. The criterion trials were presented in an uninterrupted series, and no feedback regarding accuracy was given.

\section{Results}

Average performance at each retinal location as a function of interfigure distance and relative position of the figure (central or peripheral) was calculated for each participant. The central figure was the closer of the two figures to the fixation dot for a given presentation. Central figures could appear at Retinal Locations 1, 2, or 3, and peripheral figures at Locations 3, 4, or 5, as shown in Table 1. Separate analyses of variance were carried out for figures at Location 3 and those at the other locations, since one figure always appeared at Location 3 but not at any one of the other possible locations. Visual field, distance from the other figure, and relative position were analyzed as factors.

For Location 3, the only statistically reliable effect was relative position $[\mathrm{F}(1,7)=19.98, \mathrm{p}<.01]$, with the proportion of correct responses averaging .798 for peripheral figures but only .664 for central ones. No other main effect and no interaction was significant (all ps $>.10$ ). For the analysis including all other positions, no main effect and no interaction was significant (all ps $>.10$ ).

\section{Discussion}

The asymmetry in identification accuracy consistently found with letter stimuli containing multiple distinguishing features was observed with the rotated Cs in the current experiment. This finding suggests that feature perturbations are not necessary for the asymmetry to occur. Of course, the possibility remains that a $\mathrm{C}$ does have multiple basic features, some of which may undergo perturbation and interfere with the identification of the orientation of another $\mathrm{C}$ while allowing accurate identification of the original. Therefore, ultimate tests of any feature perturbation theory must await a determination of which aspects of a letter enter the identification process as basic features.

Although most other studies of parafoveally presented stimuli have found main effects of visual field or an interaction between visual field and relative position (Krumhansl, 1977; Krumhansl \& Thomas, 1977; White, 1976), neither were found in the current experiment. As rotated Cs are probably not processed as letters (and they were not described as such), the lack of these effects is not surprising. The left-toright processing bias usually observed with letter stimuli does not often occur with nonletter figures (Bryden, 1960; but see Marzi, Di Stefano, Tassinari, \& Crea, 1979). More surprising might be the lack of an effect of interfigure distance for any stimuli. However, Krumhansl and Thomas (1977) mentioned no effect of interletter distance for targets at Location 3, although performance was poorer at Retinal Locations 2 and 4 than at Locations 1 and 5. Nevertheless, in their study no masking figure was included to the periphery of Location 5, whereas in this experiment an outer masking figure was shown which was never replaced by a stimulus figure. Thus, the level of performance at Location 5 in the earlier study may have been superior to that in this experiment. Banks, Larson, and Prinzmetal (1979) observed better performance with an interfigure distance of $1 \mathrm{deg}$ than with one of $1 / 2 \mathrm{deg}$, although this effect is apparent only with targets located 5 deg from fixation.

\section{EXPERIMENT 2}

While feature perturbations may not be involved in the performance asymmetry with the rotated Cs, the figure pairs presented in Experiment 1 contain the confounding described by Banks, Bachrach, and 
Larson (1977): With a figure in Location 4 or Location 5, the stimulus configuration as a whole was more peripheral than when a figure appeared in Location 1 or Location 2. Banks et al. (1977) interpreted their results to mean that the retinal acuity at the midpoint of the stimulus pair determines performance. While those experiments presented stimuli in the same manner as in the current experiment (so the retinal position of one figure did not vary with the position of the second), a mathematical correction for retinal sensitivity yielded equal performance for peripheral and central letters. The authors did not merely present the letter pair in a constant position to avoid the confounding of target position and configuration midpoint. If that had been done, the target would have been further from the fixation point when the second figure was on the inside than when it was on the outside. The importance that the authors ascribed to this fact, as well as their use of a mathematical correction for the array midpoint while holding retinal position constant, suggests that if the configuration were actually kept at a constant distance from fixation, performance might be no better for the peripheral than for the central figure. This would be true were the correction for the midpoint of the configuration not as effective as moving the retinal location of the target one place inward.

The current experiment was designed to determine whether parafoveal identification asymmetry is based upon the retinal sensitivity at the midpoint of the projection for the figure pair. Since the configuration was placed at a constant distance from fixation, the asymmetry should disappear to the extent of its dependence upon the proposed acuity factor.

\section{Method}

Participants. Ten students, each reporting normal or correctedto-normal vision, were given extra credit in a general psychology course for their participation in the 45-min session. None had participated in Experiment 1.

Apparatus. The apparatus and luminance settings were identical to those in the preceding experiment.

Stimuli. The masking array consisted of four dollar signs, two on each side of a central fixation dot. The dollar signs were identical in height and width to those in the preceding experiment, and members of each lateral pair were separated by a visual angle of $.268 \mathrm{deg}$. The inside edge of each innermost dollar sign lay $2.030 \mathrm{deg}$ from the center of the fixation dot, which subtended a visual angle of $.161 \mathrm{deg}$. Rotated Cs were identical to those in the preceding experiment, and were positioned in the same relationship to mask as described there. The space between the rotated $\mathrm{Cs}$ was $.108 \mathrm{deg}$ wide. The two stimulus figures appearing on each presentation were on the same slide, which was illuminated for $75 \mathrm{msec}$. Stimuli were prepared in the same manner as those used in Experiment 1, although since the contrast of the rotated $\mathrm{Cs}$ and masking figures was equal no tape covered the masking figures.

Design. Ninety-six criterion trials were given each participant, with the two rotated $\mathrm{Cs}$ appearing in each visual field on half the trials. Each $\mathrm{C}$ was rotated clockwise on half the presentations and counterclockwise on the other half. Visual field and rotation combinations were randomized with the constraint that each possible combination of variables appear twice within each block of 16 trials. The sequence was presented without interrup- tion in forward order to half the participants and in reverse order to the remainder.

Procedure. The procedure was identical to that of the preceding experiment.

\section{Results}

Average identification accuracy for each relative position within each visual field for each participant was compared in an analysis of variance. The mean effect of relative position was significant $[F(1,9)=$ $89.55, \mathrm{p}<.001$ ], with a mean proportion correct of .855 for the peripheral figure and .728 . for the central one. The other main effect and the interaction did not approach significance (both $F s<1.0$ ).

A possibility existed that the nonsensory variable of response competition might be responsible for the performance asymmetry; this was explored by reanalyzing the data with correct orientation (gap position) responses for the figure pairs included as a factor. In addition to the highly significant effect of relative position already cited, the main effect of correct response combination, as well as the interaction of correct response combination with relative position, was significant $[F(3,27)=6.71, \mathrm{p}<.01$, and $F(3,27)=7.14, p<.01$, respectively]. The nature of these effects can be seen in Figure 2. No other interaction involving response combination was significant (all ps $>.10$ ).

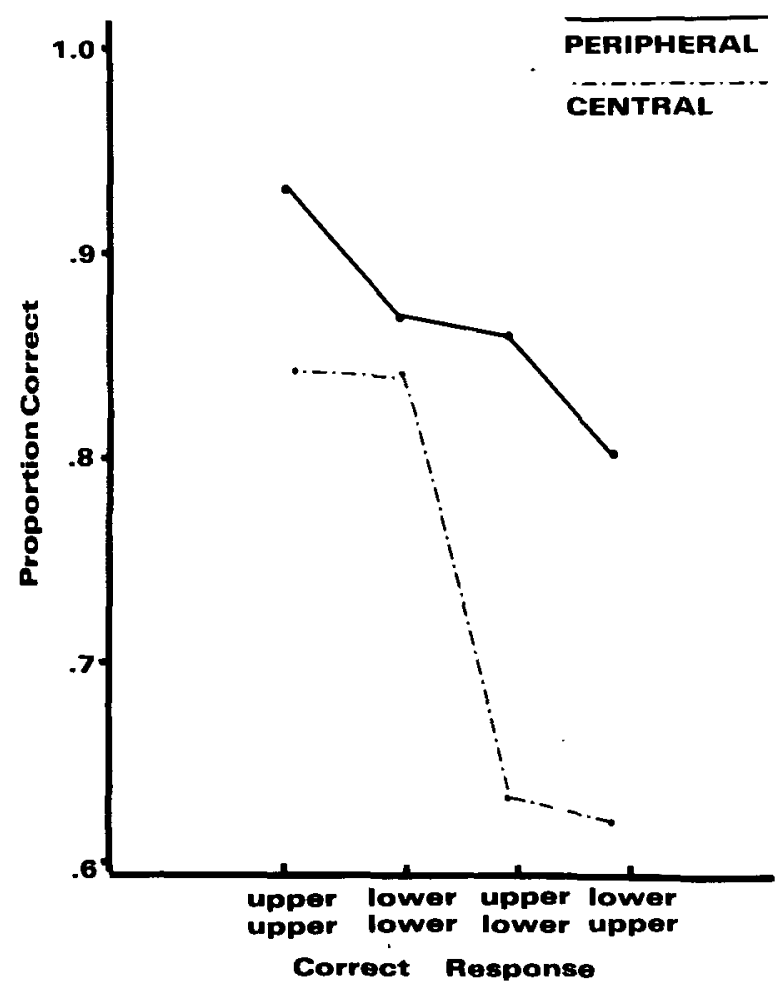

Figure 2. The relationship between response combination and accuracy of orientation identification for each relative position in Experiment 2. 


\section{Discussion}

With the stimulus configuration appearing at a constant distance from fixation, identification of the peripheral figure was markedly superior to that of the central one. Apparently the effect is not necessarily due to visual sensitivity at the midpoint of the configuration. The distance of the figure pair from fixation in the current experiment is within the range used by Banks, Bachrach, and Larson (1977), who concluded that the midpoint of the configuration was the crucial factor.

Nevertheless, in the experiment of Banks et al. (1977), the task was to identify only one member of the figure pair on each presentation, with the other member always being a letter $H$. One could hypothesize that the asymmetry noted in the current experiment resulted from some sort of processing interference such as response competition, with the central figure affected more than the peripheral one. Indeed, a reanalysis of the current results to include response combination as a variable revealed a significant interaction between this and relative position. As shown in Figure 2, most of the performance asymmetry occurred when two different words comprised the correct response for a presentation (either "upper, lower" or "lower, upper"). The result does not indicate a tendency to perseverate the response appropriate to the leftmost figure, always the first response word uttered, for no interaction occurs with visual field. Perhaps, if two different words were never required for the response, no asymmetry would be observed with the retinal position of the configuration held constant, in line with the suggestion of Banks, Bachrach, and Larson (1977), whose task required only a one-word response. The following experiment was designed to test this possibility.

\section{EXPERIMENT 3}

In this final experiment, response competition effects were eliminated by presenting only one target figure per trial. The stimulus array contained two figures, always the same distance from fixation, but only one was a rotated $\mathrm{C}$. The other closed figure resembled a rotated letter 0 . Possible decision errors from uncertainty regarding which figure was the $C$ were minimized by blocking presentations so that the central figure was the target in one block, and the peripheral figure was the target in the other.

\footnotetext{
Method

Participants. Ten students served in the experiment for extra credit in a general psychology course. Each reported having normal or corrected-to-normal vision, and each performed in a single session lasting approximately $1 \mathrm{~h}$. No participant had served in either of the two experiments previously described.

Apparatus. The apparatus and all settings were identical to those in Experiment 2. The stimulus field was again illuminated for $75 \mathrm{msec}$ per exposure.
}

Stimuli. The stimuli were identical to those in the preceding experiment, except that the gap of one rotated $C$ on each slide was filled in. This was done by a work-study student, unfamiliar with the experimental hypothesis, who inverted the letter guide so that the rear of the $C$ on the guide covered the gap in the $C$ on the stimulus card. The open area was then traced and filled in with the same Pilot Razor Point pen used to prepare the original stimuli. The filled-in area was indistinguishable in contrast and stroke width from the remainder of the figure. The mask from Experiment 2 was used in the current experiment, and alignment of the rotated $\mathrm{Cs}$ and Os with the mask was identical to that described with the figures there.

Design. Each participant was given 96 criterion trials, 48 with central targets and 48 with peripheral targets. Half the participants received the block of central targets first. Targets appeared in each visual field equally often, with the clockwise rotation on half the presentations for each visual field. Two different random orders of 48 presentations were constructed, with the constraint that each slide for either central or peripheral targets be presented four times within each block of 16 trials. Half of the participants were presented each random order in the forward sequence, and half in the reverse sequence. The $\mathbf{4 8}$ trials for the central position were presented without interruption as the first block to half of the participants, but as the second block to the remainder, and this order was analyzed as a between-subjects variable.

Procedure. The procedure was identical to that used in the preceding experiment, except that familiarization, practice (16 triais), and the 48 criterion trials were given initially with the target in only one relative position. The participant was then familiarized with the target in the other relative position, and given 16 practice trials followed by 48 criterion trials for this target position. The required verbal response after each presentation was the single word "lower" or "upper."

\section{Results}

Average identification accuracy was calculated for each orientation, target position, and visual field for each participant within each order. The resulting means were compared in an analysis of variance. The main effect of relative target position was significant $[F(1,8)=8.33, p<.025]$. The proportion of presentations yielding accurate identification was .829 for peripheral targets and .748 for central ones. No other main effect and no interaction was significant (all ps $>.10$ ). As the mean difference in accuracy between central and peripheral targets in the current experiment (.081) was smaller than that in the preceding experiment (.127), a between-groups analysis of variance was conducted in search of an Experiments by Position interaction. The interaction was not significant $[F(1,18)=1.84, p>.10]$.

\section{Discussion}

When identification of only one target was required, and retinal position of the configuration was held constant, performance on the peripheral target was superior to that on the central target. The effect emerged as slightly weaker than in the first two experiments, perhaps due to reduced reliability resulting from halving the number of trials upon which figures at a given relative position were identified. Nevertheless, the performance asymmetry remains after processing interference from decision or response factors has been minimized. 


\section{GENERAL DISCUSSION}

The purpose of the three experiments just reported was to test various explanations which have been offered for the asymmetric performance typically observed on parafoveally presented stimulus pairs such that if a figure is peripheral it is more accurately identified than if it is central. The suggestion that feature perturbations from the peripheral to the central figure leave the former identifiable while obscuring the latter can currently be tested only by making assumptions regarding the nature of basic features. If distinguishing features are basic features, feature perturbations seem unnecessary for identification asymmetry to occur. Another possibility, that the retinal sensitivity at the locus of the midpoint of the projection of the figure pair determines the effect, was unsupported by the combined results of the last two experiments. Traditionally, the figure pairs have been presented so that the retinal position of the target is constant and the second figure is peripheral or central to the target, making the entire configuration more peripheral with a central target. However, if the location of the configuration is held constant, identification of the central figure is still less accurate.

If a pair of rotated $\mathrm{Cs}$ is presented, identification of the orientation of both figures requires the utterance of two words. When the orientations differ for the figures, the average difference in performance between the central and peripheral figure is much greater than if the orientations are the same. Perhaps the interaction is due to response perseveration, not of the response to the leftmost figure, but to the most clearly visible figure, the peripheral one. When only one response is required by introducing a second figure in a constant position as a nontarget, the performance asymmetry remains, rendering response perseveration an unlikely explanation.

Banks, Larson, and Prinzmetal (1979) emphasize the point that adjacent stimuli are likely to be grouped as a Gestalt in the visual periphery (Beck, 1972). Poorer identification of a parafoveal target with a peripheral nontarget than with a central one thus can be related to the difficulty of isolating the target from the cluster, with performance predictably worse as the midpoint of the cluster moves further from the fovea. However, the peripheral figure is still identified significantly better than the central one with the mid- point of the cluster at a constant distance from fixation. A grouping explanation seems to offer no prediction that characteristics of the peripheral area of the Gestalt are more easily identified than those in the more central area.

Since none of the explanations for the performance asymmetry considered so far seems adequate, additional research is definitely implicated.

\section{REFERENCES}

Banks, W. P., Bachrach, K. M., \& Larson, D. W. The asymmetry of lateral interference in visual letter identification. Perception \& Psychophysics, 1977, 22, 232-240.

Banks, W. P., Larson, D. W., \& Prinzmetal, W. Asymmetry of visual interference. Perception \& Psychophysics, 1979, 25, 447-456.

BEck, J. Similarity grouping and peripheral discriminability under uncertainty. American Journal of Psychology, 1972, 85, $1-19$.

BRYDEN, M. P. Tachistoscopic recognition of nonalphabetic material. Canadian Journal of Psychology, 1960, 14, 78-86.

Estes, W. K., Allmeyer, D. H., \& Reder, S. M. Serial position functions for letter identification at brief and extended exposure durations. Perception \& Psychophysics, 1976, 19, 1-15.

Gibson, E. J. Principles of perceptual learning and development. New York: Appleton-Century-Crofts, 1969.

KrumhansL, C. L. Naming and locating simultaneously and sequentially presented letters. Perception \& Psychophysics, 1977, 22, 293-302.

Krumhansl, C. L., \& Thomas, E. A. C. Effect of level of confusability on reporting letters from briefly presented visual arrays. Perception \& Psychophysics, 1977, 21, 269-279.

Lindsay, P. H., \& Norman, D. A. Human information processing. New York: Academic Press, 1972.

LUPKER, S. J. On the nature of perceptual information during letter perception. Perception \& Psychophysics, 1979, 25, 303-312.

Marzi, C. A., Di Stefano, M., Tassinari, G., \& Crea, F. Iconic storage in the two hemispheres. Journal of Experimental Psychology: Human Perception and Performance, 1979, 5, 31-41.

TAylor, S. G., \& Brown, D. R. Lateral visual masking: Supraretinal effects when viewing linear arrays with unlimited viewing time. Perception \& Psychophysics, 1972, 12, 97-99.

Townsend, J. T. Theoretical analysis of an alphabetic confusion matrix. Perception \& Psychophysics, 1971, 9, 40-50.

Townsend, J. T., TAylor, S. G., \& Brown, D. R. Lateral masking for letters with unlimited viewing time. Perception \& Psychophysics, 1971, 10, 375-378.

Whiтe, M. J. Order of processing in visual perception. Canadian Journal of Psychology, 1976, 30, 140-156.

Wolford, G. Perturbation model for letter identification. Psychological Review, 1975, 82, 184-199.

(Received for publication July 10, 1979; revision accepted September 20, 1979.) 